\title{
Relaxamento Guiado como Prática Integrativa para Mulheres Submetidas à Radioterapia
}

doi: https://doi.org/10.32635/2176-9745.RBC.2022v68n1.1326

\author{
Guided Relaxation as an Integrative Practice for Women Undergoing Radiation Therapy \\ Relajación Guiada como Práctica Integradora para Mujeres Sometidas a Radioterapia
}

Lais Reis Siqueira'; Sterline Therrier²; Kamilla Maestá Agostinho³; Edison Vitório de Souza Júnior; Zélia Marilda Rodrigues Resck5; Namie Okino Sawada ${ }^{6}$

RESUMO

Introduçáo: $\mathrm{O}$ câncer de mama ocupa uma das primeiras posiçóes das neoplasias malignas em mulheres. Entre os tipos de tratamento, está a radioterapia que, apesar de ser um método seguro, traz diversos efeitos colaterais que prejudicam a qualidade de vida relacionada à saúde. Objetivo: Avaliar o efeito da prática integrativa e complementar de relaxamento com visualizaçáo guiada na melhora da qualidade de vida relacionada à saúde de mulheres com câncer de mama submetidas à radioterapia. Método: Pesquisa quantitativa, quase-experimental, do tipo pré e pós-teste, realizada com 25 mulheres com câncer de mama submetidas à radioterapia em um Centro de Oncologia do Sul de Minas Gerais, entre julho de 2019 a março de 2020. Foram coletados dados sobre aspectos sociodemográficos e clínicos, e utilizado o instrumento Quality of Life Questionnaire-Core $30 \mathrm{da}$ European Organization for Research and Treatment of Cancer (EORTC QLQ-C30) para avaliação da qualidade de vida, aplicado em três momentos do tratamento. As seções de relaxamento foram aplicadas três vezes na semana durante todo o tratamento radioterápico. Resultados: A maioria das pacientes se encontrava na faixa etária de 41-60 anos, com nível médio de escolaridade, aposentadas, católicas, brancas, casadas e em estadiamento IIA. Os escores relacionados às escalas funcionais apresentaram melhora ao longo do tratamento. Para a escala de sintomas, os prevalentes foram insônia, constipação e fadiga. Conclusáo: A prática de relaxamento foi eficaz na melhora dos domínios da qualidade de vida relacionada à saúde, sendo uma prática de baixo custo que pode ser aplicada por profissionais treinados.

Palavras-chave: neoplasias da mama/radioterapia; qualidade de vida; terapia de relaxamento; terapias complementares.

\section{ABSTRACT}

Introduction: Breast cancer is one of the leading malignant neoplasms in women. Radiotherapy is among the types of treatment which, although safe, causes several side effects that impair health-related quality of life. Objective: To evaluate the effect of integrative and complementary practice of relaxation with guided visualization in improving health-related quality of in women with breast cancer undergoing radiotherapy. Method: Quantitative, quasi-experimental, pre-and post-test study, conducted with 25 women with breast cancer undergoing radiotherapy at an Oncology Center in the South of Minas Gerais, between July 2019 and March 2020. Sociodemographic and clinical data were collected, the Quality-of-Life Questionnaire-Core 30 of the European Organization for Research and Treatment of Cancer (EORTC QLQ-C30) was used to assess quality of life, applied at three stages of the treatment. The relaxation sections were applied three times a week during the entire radiotherapy treatment. Results: Most of the patients were aged 41-60 years, with an average level of education, retired, Catholic, white, married and in IIA staging. The scores related to the functional scales showed improvement throughout the treatment. The prevalent symptoms for the symptom scale were insomnia, constipation and fatigue. Conclusion: The practice of relaxation was effective in improving the domains of health-related quality of life, being a low-cost practice that can be applied by trained professionals.

Key words: breast neoplasms/radiotherapy; quality of life; relaxation therapy; complementary therapies.

\section{RESUMEN}

Introducción: El cáncer de mama ocupa una de las primeras posiciones de las neoplasias malignas en la mujer. Entre los tipos de tratamiento se encuentra la radioterapia que, a pesar de ser un método seguro, tiene varios efectos secundarios que perjudican la calidad de vida relacionada con la salud. Objetivo: Evaluar el efecto de la práctica integradora y complementaria de la relajación con visualización guiada en la mejora de la calidad de vida relacionada con la salud de mujeres con cáncer de mama sometidas a radioterapia. Método: Cuantitativo, cuasiexperimental, tipo pre y posprueba, realizado con 25 mujeres con cáncer de mama sometidas a radioterapia en un Centro de Oncología del Sur de Minas Gerais, entre julio de 2019 y marzo de 2020 Datos sociodemográficos y clínicos Se recogieron aspectos y se utilizó el instrumento Quality of Life QuestionnaireCore 30 de la Organización Europea para la Investigación y el Tratamiento del Cáncer (EORTC QLQ-C30) para evaluar la calidad de vida, aplicado en tres etapas del tratamiento. Las secciones de relajación se aplicaron tres veces por semana durante todo el tratamiento de radioterapia. Resultados: La mayoría de los pacientes tenían entre 41 y 60 ańos, con nivel educativo medio, jubilados, católicos, blancos, casados y en estadificación IIA. Las puntuaciones relacionadas con las escalas funcionales mostraron mejoría a lo largo del tratamiento. Los síntomas prevalentes para la escala de síntomas fueron insomnio, estreñimiento y fatiga. Conclusión: la práctica de la relajación resultó efectiva para mejorar los dominios de la calidad de vida relacionada con la salud, siendo una práctica de bajo costo que puede ser aplicada por profesionales capacitados.

Palabras clave: neoplasias de la mama/radioterapia; calidad de vida; terapia por relajación; terapias complementarias.

\footnotetext{
1,2,5,6Universidade Federal de Alfenas (Unifal). Alfenas (MG), Brasil. E-mails: laisreis.siqueira@gmail.com; sterlinetherrier@gmail.com; zmrresck57@gmail.com; namie.sawada@unifal-mg.edu.br. Orcid iD: https://orcid.org/0000-0002-6720-7642; Orcid iD: https://orcid.org/0000-0002-7713-3288; Orcid iD: https://orcid. org/0000-0002-3752-8381; Orcid iD: http://orcid.org/0000-0002-1874-3481

3,4Universidade de São Paulo (USP). Escola de Enfermagem de Ribeirão Preto (EERP). Ribeirão Preto (SP), Brasil. E-mails: kamillamaesta@gmail.com; edison.vitorio@usp.br. Orcid iD: https://orcid.org/0000-0001-8377-2329; Orcid iD: http://orcid.org/0000-0003-0457-0513

Endereço para correspondência: Lais Reis Siqueira. Unifal. Rua Gabriel Monteiro da Silva, 700 - Parque das Nações. Alfenas (MG), Brasil. CEP $37130-001$. E-mail: laisreis.siqueira@gmail.com
} 


\section{INTRODUÇÃO}

O câncer de mama aparece como o tipo mais comumente diagnosticado entre as mulheres depois do câncer de pele não melanoma. Foram registrados 2,1 milhóes de novos casos em 2018. Constituiu-se a primeira causa de mortalidade por câncer em mulheres em todo o mundo, com registro de 627 mil mortes em 2018 ${ }^{1}$.

Para o Brasil, estimam-se, para cada ano do triênio 2020-2022, 625 mil casos novos de câncer, sendo o de pele não melanoma o mais incidente, seguido pelo câncer de próstata nos homens e mama nas mulheres. $\mathrm{O}$ câncer de mama apresenta um risco estimado de 61,61 casos novos a cada 100 mil mulheres ${ }^{2}$.

O tipo de tratamento é escolhido a depender de fatores como estadiamento do câncer, idade, condiçôes clínicas, fatores de risco, critérios histopatológicos, biológicos, moleculares e genéticos ${ }^{3,4}$. Entre os tratamentos para o câncer de mama, podem ser incluídos a mastectomia radical ou conservadora, quimioterapia, hormonioterapia, terapia biológica e a radioterapia ${ }^{5,6}$.

Inicialmente, a mastectomia radical caracterizava o tratamento cirúrgico do câncer de mama. Essa conduta passou a ser questionada com os resultados de pesquisas que evidenciavam haver resultados de sobrevida semelhantes com a indicaçáo da cirurgia conservadora associada à radioterapia ${ }^{7,8}$.

Em decorrência do aumento das indicaçóes para cirurgias conservadoras, elevaram-se as recomendaçôes de radioterapia para o período pós-operatório, com intuito de reduzir riscos de recorrência locorregional ${ }^{9}$.

Entre os tipos de radioterapia, podem ser citadas a teleterapia, sendo a mais comumente utilizada, na qual a fonte de radiação está afastada do paciente; e a braquiterapia, na qual são implantados aplicadores em contato com a área a ser irradiada ${ }^{10,11}$. A braquiterapia é pouco utilizada para o câncer de mama, visto que seu resultado estético não é favorável ${ }^{12}$.

Apesar dos avanços tecnológicos, a radioterapia para o câncer de mama ainda apresenta diversos efeitos colaterais desfavoráveis, podendo ser classificados em imediatos, sendo os mais recorrentes a radiodermatite, o escurecimento da pele, prurido, descamação, dor local, desconforto axilar e a fadiga ${ }^{13,14}$. As complicaçóes tardias compreendem os problemas cardíacos associados com irradiação da regiáo torácica, lesōes pulmonares, problemas no sistema endócrino e restrição da movimentação dos braços, em virtude da irradiação da área onde foi realizada a cirurgia ${ }^{15,16}$.

Neste sentido, a avaliação da qualidade de vida relacionada à saúde (QVRS), em pacientes em tratamentos oncológicos, tornou-se essencial, pois, a partir do conhecimento sobre os efeitos colaterais relacionados ao tratamento, torna-se possível a criação estratégias capazes de amenizar os prejuízos causados aos domínios da qualidade de vida ${ }^{17}$.

O termo QVRS tem a intenção de quantificar as consequências de uma enfermidade e de seus tratamentos, de acordo com a percepção que o indivíduo possui sobre a sua capacidade para ter uma vida útil e desenvolver suas atividades ${ }^{18}$.

Em razão das comprovaçóes do aumento da sintomatologia e da consequente redução da QVRS decorrentes das terapias oncológicas, surgem as práticas integrativas e complementares em Saúde (PICS) com intençáo de apoiar a terapia convencional, reduzindo efeitos colaterais, melhorando a QVRS e aumentando a adesáo ao tratamento. Neste cenário, as mulheres constituem o maior público de usuários das PICS em nível nacional e internacional ${ }^{19}$.

As diversas PICS vêm se destacando por causa da sua ampla utilizaçáo em pacientes com câncer em uso de terapias oncológicas, objetivando reduzir sintomas de dor, fadiga, ansiedade, depressão, náusea e vômitos e consequentemente melhorar o autocuidado e a QVRS ${ }^{20-23}$.

Neste estudo, a PICS utilizada foi o relaxamento com visualização guiada que consiste em uma prática mente-corpo, a qual se concentra nas interaçóes entre cérebro, mente, corpo e comportamento, e na maneira como os fatores emocionais, mentais e comportamentais afetam a saúde ${ }^{24}$.

Pela Portaria $\mathrm{n}^{\circ} 702^{25}$, de 21 de março de 2018, foram incluídas novas PICS na Política Nacional de Práticas Integrativas e Complementares (PNPIC), a exemplo da aromaterapia, apiterapia, bioenergética, constelação familiar, cromoterapia, geoterapia, hipnoterapia, imposição de mãos, medicina antroposófica/antroposofia aplicada à saúde, ozonioterapia, terapia de florais e crenoterapia. $\mathrm{O}$ relaxamento com visualização ainda não se insere como uma técnica ofertada pelo Sistema Único de Saúde (SUS).

No entanto, o profissional enfermeiro sendo detentor de conhecimento e o profissional que passa maior parte do tempo próximo ao paciente, deve buscar constantemente técnicas que necessitam de poucos recursos tecnológicos e de baixo custo, mas que sejam capazes de trazer benefícios a esse público fragilizado.

A técnica de relaxamento com visualização guiada enquadra-se nas PICS, e vem sendo amplamente utilizada como estratégia de intervenção da enfermagem, demonstrando ser uma prática efetiva para o controle e a redução dos sintomas decorrentes do câncer e de seu tratamento.

Com isso, o objetivo deste estudo foi avaliar o efeito da PICS de relaxamento com visualização guiada na 
melhora da QVRS de mulheres com câncer de mama em radioterapia.

\section{MÉTODO}

Estudo com abordagem quantitativa, quase-experimental, com avaliação pré e pós-teste. Realizando-se a coleta de dados em um setor de radioterapia de um Centro de Oncologia do Sul de Minas Gerais, entre julho de 2019 a março de 2020. A seleção da amostra foi realizada por conveniência. Foram incluídas mulheres com idade igual ou superior a 18 anos, diagnosticadas com câncer de mama que estavam iniciando o tratamento radioterápico.

Foram excluídas as pacientes que não apresentassem condiçôes clínicas e cognitivas que possibilitassem responder aos questionários e participar do relaxamento. A avaliação cognitiva para seleção das participantes foi realizada mediante os seguintes questionamentos: nome completo, dia da semana, data de nascimento, endereço. Excluíram-se, também, pacientes que estivessem participando de outro tipo de terapia integrativa e complementar no momento ou estivessem em tratamento quimioterápico concomitante com o radioterápico, em razáo da possibilidade de exacerbação de efeitos colaterais.

Como critérios de descontinuidade, foram considerados o náo comparecimento para as sessóes de relaxamento (mínimo em oito sessóes de relaxamento a depender do número de sessôes de radioterapia realizado), desistência por parte da participante e complicaçóes relacionadas ao tratamento. Inicialmente, considerando os critérios de inclusão e exclusão, 19 participantes não atendiam a esses critérios, 12 por estarem realizando quimioterapia concomitante à radioterapia, três por não apresentarem condiçôes clínicas e cognitivas e quatro que se recusaram a participar da pesquisa. Desta maneira, foram selecionadas inicialmente 30 participantes, sendo que uma participante apresentou dificuldade com o transporte impossibilitando a participação em todas as sessôes requeridas, uma participante relatou desistência por dificuldades de concentração durante as sessóes de relaxamento e uma paciente apresentou radiodermite, necessitando de interrupção do tratamento radioterápico.

Primeiramente, foi realizado um levantamento semanal das pacientes que estavam iniciando tratamento radioterápico, estas foram abordadas e explicados os objetivos da pesquisa, sendo a amostra selecionada pela técnica consecutiva náo probabilística. As que aceitaram participar da pesquisa assinaram o Termo de Consentimento Livre e Esclarecido.

Em seguida, foram encaminhadas individualmente para um local privativo, posicionadas em uma poltrona confortável com apoio para os pés, onde responderam ao questionário sociodemográfico e clínico terapêutico e ao instrumento que avaliou a QVRS - o Quality of Life Questionnaire-Core 30 (QLQ-C30) da European Organization for Research and Treatment of Cancer (EORTC), validado para população brasileira ${ }^{26}$, para uso específico em pacientes com câncer.

As questôes foram direcionadas para que os informes estivessem relacionados com a última semana da radioterapia. As respostas seguem pontuação de escala do tipo Likert. De acordo com diretrizes do EORTC, essas escalas e itens geram escores que são transformados em uma escala de 0 a 100 , na qual zero denota o pior funcionamento e 100, o melhor funcionamento nas escalas funcionais e estado geral de saúde/qualidade de vida (EGS/QV), enquanto, nas escalas e itens de sintomas e no item de impacto financeiro, ocorre o inverso, quanto mais próximo de zero, indica menos sintomas presentes e, quanto mais próximo de 100 , mais sintomas ${ }^{27}$.

Após responderem aos questionários e se declararem confortáveis, foi iniciada a sessão de relaxamento, guiada por um $\mathrm{CD}$, produzido pelo centro de apoio ao paciente com câncer de Florianópolis, SC, com duração de aproximadamente 15 minutos, onde as pacientes eram orientadas a fechar os olhos e iniciar o processo de relaxamento por meio de movimentos respiratórios profundos, imaginado cenas agradáveis da natureza, como o canto dos pássaros e o som do mar. Em seguida, cada paciente foi conduzida a criar em sua mente a imagem do seu câncer e seus glóbulos brancos, combatendo-o, e seu corpo forte e sadio novamente. Além disso, a participante foi convidada a visualizar cenas relacionadas com atividades que achava que não poderia fazer por causa da doença, criando expectativa e confiança de que agora poderá realizá-las. Ao final, solicitou-se a paciente que agradecesse, por ter dado esse tempo a si mesma, e que abrisse os olhos devagar retornando ao ambiente.

O instrumento EORTC QLQ-C30 foi aplicado em três momentos do tratamento radioterápico em T1 (baseline) quando essas mulheres estavam iniciando o tratamento radioterápico, considerada até a quarta sessão de radioterapia, T2 (meio) e T3 (final) do tratamento radioterápico. As sessóes de relaxamento foram aplicadas por três vezes na semana durante todo o período de radioterapia.

Para análise dos dados, foi utilizado software Statistical Package for Social Science versão 24.0 (SPSS for Windows). Foram calculados médias e desvio-padrão para análise descritiva. Nas descriçóes dos comportamentos das variáveis tomadas duas a duas, foi empregado o teste de Wilcoxon, para evidenciar diferenças significativas $(p<0,05)$. 
A pesquisa foi aprovada pelo Comitê de Ética em Pesquisa da Universidade Federal de Alfenas (CEP/ Unifal-MG), sob parecer número 3.334.033.

\section{RESULTADOS}

Participaram do estudo 27 mulheres com câncer de mama em tratamento radioterápico selecionadas segundo os critérios de inclusão.

Em relação às características sociodemográficas, a maioria estava na faixa etária de 41-60 anos (44\%), casadas $(63 \%)$, aposentadas/donas de casa $(37 \%)$, variando entre outras profissóes como: professora, caminhoneira, assistente financeira, empregada doméstica e auxiliar de limpeza. Com nível médio de escolaridade (63\%), brancas (56\%), e procedentes de cidades do Sul de Minas Gerais (96\%).

Quanto ao número de filhos, 77\% possuíam dois filhos ou mais e, em relação ao tempo de amamentação, 56\% haviam amamentado por mais de seis meses.

Em relação aos procedimentos terapêuticos, 96\% passaram por procedimento cirúrgico, sendo que $30 \%$ realizaram apenas a retirada de nódulo, 33\% quadrantectomia e 33\% mastectomia total. Em relaçáo ao número de sessões de radioterapia, $81 \%$ haviam realizado entre 18 a 20 sessôes e $19 \%$ realizaram de 20 até 30 sessōes.
Destas, apenas 30\% não haviam realizado quimioterapia anterior à radioterapia.

Com relação às características psicométricas do instrumento QLQ-C30, foi calculado coeficiente Alpha de Cronbach para T1 $\alpha=0,815$, T2 $\alpha=0,816$ e T3 $\alpha=0,888$, indicando confiabilidade do instrumento nos três tempos.

A Tabela 1 mostra a média e o desvio-padrão das escalas do QLQ-C30 nos três tempos de avaliação.

Por meio da análise descritiva do questionário EORTC QLQ-C30, na Tabela 1, é possível verificar que os escores iniciais da escala funcional classificam-se com um nível satisfatório. Nota-se que, nas consecutivas avaliaçóes em T2 e T3, o EGS/QV e as cinco escalas funcionais apresentaram um nível satisfatório, porém com aumento nas médias de escore, podendo ser inferido que esses domínios melhoraram ao longo do tratamento radioterápico.

Em relação aos escores iniciais da escala de sinais e sintomas, verifica-se que as sintomatologias mais frequentes foram insônia, constipação, fadiga e dor. Esses escores se mantiveram inalterados ou melhoraram ao longo do tratamento radioterápico. A fadiga, dor e constipação reduziram consecutivamente. No entanto, o escore relacionado com a insônia apresentou maior escore em relação ao inicial em T2, mas em T3 retornou ao apresentado inicialmente, e o escore perda de apetite

Tabela 1. Média e desvio-padrão das escalas do instrumento QLQ-C30, nos três tempos de avaliação, T1 (baseline) T2 (meio) e T3 (final), Alfenas, MG, 2019-2020

\begin{tabular}{cccc}
\hline Escalas & T1 & T2 & T3 \\
\cline { 2 - 4 } & Média (DP) & Média (DP) & Média (DP) \\
\hline Estado geral de saúde & $76(24)$ & $81(19)$ & $87(14)$ \\
Função física & $77(22)$ & $81(19)$ & $83(19)$ \\
Desempenho de papel & $77(30)$ & $78(25)$ & $87(24)$ \\
Função cognitiva & $78(24)$ & $81(22)$ & $84(20)$ \\
Função social & $86(17)$ & $84(22)$ & $85(24)$ \\
Função emocional & $77(26)$ & $77(21)$ & $83(23)$ \\
Escala de sintomas & & & \\
Fadiga & $21(21)$ & $19(17)$ & $18(19)$ \\
Náuseas e vômitos & $7(18)$ & $6(11)$ & $3(10)$ \\
Dor & $17(25)$ & $13(22)$ & $13(16)$ \\
Dispneia & $11(23)$ & $10(22)$ & $11(18)$ \\
Insônia & $33(43)$ & $36(43)$ & $33(43)$ \\
Perda de apetite & $16(28)$ & $18(30)$ & $9(22)$ \\
Constipação & $22(33)$ & $21(31)$ & $21(31)$ \\
Diarreia & $2(9)$ & $2(9)$ & $0(0)$ \\
Dificuldades financeiras & $14(27)$ & $4(11)$ & $6(16)$
\end{tabular}

Legenda: $\mathrm{DP}=$ Desvio-padrăo. 
que também apresentou escore mais elevado em T2, apresentando redução de escore em T3.

Não houve deterioração significativa de nenhuma escala, ao final do tratamento radioterápico. Inclusive, todas escalas funcionais e EGS/QV mantiveram-se como satisfatórias.

Visando a comparar os domínios de QVRS entre as avaliaçóes nos três tempos, a Tabela 2 apresenta o Teste de Wilcoxon das escalas do QLQ-C30 entre os tempos T1(baseline), T2 (meio) e T3 (final), sendo demonstrado a seguir pelas Tabelas 2, 3 e 4 .

$\mathrm{Na}$ Tabela 2, foram comparadas a avaliação inicial e a avaliação intermediária do tratamento radioterápico, ou seja, de ( $T 1 \times$ x $T 2)$. Nota-se que não foram demonstradas diferenças estatisticamente significativas entre esses tempos.

Tabela 2. Teste de Wilcoxon do instrumento QLQ-C30, entre os tempos de $(T 1 \times T 2)$, Alfenas, MG, 2019-2020

\begin{tabular}{ccc}
\hline Variáveis & $\mathbf{z}$ & $\boldsymbol{p}$ \\
\hline EGS/QV $\times$ EGS/QV & 1,064 & 0,1437 \\
FF $\times$ FF & 1,549 & 0,0607 \\
DP $\times$ DP & 0,207 & 0,4181 \\
FC $\times$ FC & 1,255 & 0,1046 \\
FS $\times$ FS & 0,420 & 0,3372 \\
FE $\times$ FE & 0,402 & 0,3437 \\
FAD $\times$ FAD & 0,544 & 0,2931 \\
NAV $\times$ NAV & 0,314 & 0,3766 \\
DOR $\times$ DOR & 1,067 & 0,1430 \\
DIS $\times$ DIS & 0,269 & 0,3937 \\
INS $\times$ INS & 0,629 & 0,2647 \\
PAP $\times$ PAP & 0,350 & 0,3631 \\
CON $\times$ CON & 0,255 & 0,3994 \\
DIA $\times$ DIA & 0,000 & 0,5000 \\
DIF $\times$ DIF & 2,201 & 0,0139 \\
\hline
\end{tabular}

Legendas: $\mathrm{EGS} / \mathrm{QV}=$ Estado geral de saúde/qualidade de vida; $\mathrm{FF}=$ Função física; $\mathrm{DP}=$ Desempenho de papel; $\mathrm{FC}=$ Funçăo cognitiva; FS = Funçăo social; $\mathrm{FE}=$ Funçáo emocional; FAD = Fadiga; NAV = Náuseas/vômitos; $\mathrm{DOR}=$ Dor; DIS = Dispneia; INS = Insônia; PAP = Perda de apetite; CON = Constipação; DIA $=$ Diarreia; DIF $=$ Dificuldades financeiras .

Na Tabela 3, de T2 para T3, as diferenças estatisticamente significantes foram encontradas para as escalas de desempenho de papel $(p=0,0119)$ e função emocional $(p=0,0115)$, indicando que a intervenção contribuiu na melhora desses domínios.

$\mathrm{Na}$ Tabela 4, as diferenças estatisticamente significantes foram encontradas de T1 para T3 no EGS/QV $(p=0,0062)$ nas escalas de função física $(p=0,0360)$, desempenho de papel $(p=0,0450)$, função emocional $(p=0,0166)$, insônia $(p<0,0001)$ e dificuldade financeira $(p=0,0467)$.
Tabela 3. Teste de Wilcoxon do instrumento QLQ-C30, entre os tempos de (T2×T3), Alfenas, MG, 2019-2020

\begin{tabular}{ccc}
\hline Variáveis & $\mathbf{z}$ & $\boldsymbol{p}$ \\
\hline EGS/QV $\times$ EGS/QV & 1,642 & 0,0502 \\
FF $\times$ FF & 1,363 & 0,0865 \\
DP $\times$ DP & 2,260 & $0,011 *^{*}$ \\
FC $\times$ FC & 1,121 & 0,1311 \\
FS $\times$ FS & 0,269 & 0,3937 \\
FE $\times$ FE & 2,274 & $0,0115^{*}$ \\
FAD $\times$ FAD & 0,560 & 0,2847 \\
NAV $\times$ NAV & 0,944 & 0,1726 \\
DOR $\times$ DOR & 0,722 & 0,2352 \\
DIS $\times$ DIS & 0,269 & 0,3937 \\
INS $\times$ INS & 0,629 & 0,2647 \\
PAP $\times$ PAP & 1,478 & 0,0697 \\
CON $\times$ CON & 0,000 & 0,5000 \\
DIA $\times$ DIA & 0,000 & 0,0899 \\
DIF $\times$ DIF & 1,342 & 0,0899 \\
\hline
\end{tabular}

Legendas: $\mathrm{EGS} / \mathrm{QV}=$ Estado geral de saúde/qualidade de vida; $\mathrm{FF}=$ Funçăo física; DP = Desempenho de papel; FC = Funçấo cognitiva; FS = Funçáo social; $\mathrm{FE}=$ Funçâo emocional; FAD = Fadiga; $\mathrm{NAV}=$ Náuseas/vômitos; $\mathrm{DOR}=$ Dor; DIS = Dispneia; INS = Insônia; PAP = Perda de apetite; $\mathrm{CON}=$ Constipação; DIA $=$ Diarreia DIF $=$ Dificuldades financeiras. $\left({ }^{*}\right) \mathrm{p}<0,05$.

Tabela 4. Teste de Wilcoxon do instrumento QLQ-C30, entre os tempos de (T1×T3), Alfenas, 2019-2020

\begin{tabular}{ccc}
\hline Variáveis & $\mathbf{z}$ & $\boldsymbol{p}$ \\
\hline EGS/QV $\times$ EGS/QV & 2,499 & $0,0062^{*}$ \\
FF $\times$ FF & 1,799 & $0,0360^{*}$ \\
DP $\times$ DP & 1,695 & $0,0450^{*}$ \\
FC $\times$ FC & 1,645 & 0,0500 \\
FS $\times$ FS & 0,000 & 0,5000 \\
FE $\times$ FE & 2,130 & $0,0166^{*}$ \\
FAD $\times$ FAD & 1,088 & 0,1384 \\
NAV $\times$ NAV & 0,809 & 0,2092 \\
DOR $\times$ DOR & 0,282 & 0,3888 \\
DIS $\times$ DIS & 0,000 & 0,5000 \\
INS $\times$ INS & 0,917 & 0,1795 \\
PAP $\times$ PAP & 1,244 & 0,1068 \\
CON $\times$ CON & 0,051 & 0,4797 \\
DIA $\times$ DIA & 1,342 & 0,0899 \\
DIF $\times$ DIF & 1,677 & $0,0467^{*}$ \\
\hline
\end{tabular}

Legendas: $\mathrm{EGS} / \mathrm{QV}=$ Estado geral de saúde/qualidade de vida; $\mathrm{FF}=$ Funçáo física; $\mathrm{DP}=$ Desempenho de papel; FC = Função cognitiva; FS = Funçăo social; $\mathrm{FE}=$ Função emocional $; \mathrm{FAD}=$ Fadiga $; \mathrm{NAV}=$ Náuseas/vômitos; $\mathrm{DOR}=\mathrm{Dor}$; DIS = Dispneia; INS = Insônia; PAP = Perda de apetite; CON = Constipaçăo; DIA $=$ Diarreia $;$ DIF $=$ Dificuldades financeiras.

$\left({ }^{*}\right) \mathrm{p}<0,05$. 


\section{DISCUSSÃO}

Conhecer o perfil sociodemográfico e clínico terapêutico faz-se essencial quando se pretende aplicar uma intervençâo e investigar seus efeitos sobre a QVRS. Neste estudo, a classe de maior frequência para idade foi a de 41-60 anos. Constatando-se que a incidência do câncer de mama tende a aumentar com a idade, especialmente acima dos 50 anos, fato que corrobora os dados de outros estudos ${ }^{9}$. Porém, tem-se observado um aumento dessa incidência entre mulheres mais jovens nos últimos anos. No Brasil, mulheres com menos de 35 anos apresentam uma incidência de $4 \%$ a $5 \%$, sendo anteriormente representada por apenas $2 \%$. Essas condiçóes também vêm sendo observadas em outros países em desenvolvimento, podendo esse fato ser explicado pelas mudanças no estilo de vida, que se tornaram mais comuns nos dias atuais, como menor número de filhos, gestação tardia e alimentação inadequada'.

Em relação ao estado civil, outros estudos desenvolvidos com mulheres em tratamentos oncológicos demonstraram serem a maioria casadas ${ }^{28,29}$.

Quanto às profissôes apresentadas pelas participantes deste estudo, estáo em acordo com nível de escolaridade descrito por elas, boa parte das pacientes apresentaram nível médio e demonstravam possuir conhecimento em relação ao processo patológico e aos efeitos colaterais advindos do tratamento. É referido que possuir baixa escolaridade pode dificultar o acesso às informaçóes de qualidade $^{30}$, e que o conhecimento em relaçáo ao câncer de mama e seus fatores de risco aumentam à medida que a escolaridade aumenta ${ }^{31}$.

No que concerne à cor, a maioria das mulheres se autodeclararam de cor branca. Apesar de ser um tipo de câncer comum em todas as etnias, ocorre com maior frequência em mulheres brancas, sendo um de seus fatores mais bem estabelecidos ${ }^{9,11}$. A maior parte das participantes declararam ser católicas, o que vai ao encontro da literatura, cuja principal crença religiosa em mulheres com câncer em terapias oncológicas foi o catolicismo ${ }^{32,33}$. A espiritualidade e a religiosidade se destacam como fatores contribuintes no processo de recuperação da saúde e enfrentamento da doença, possibilitando um novo sentido à experiência do adoecimento ${ }^{34}$.

Quanto ao número de filhos, a maior parte possuía até dois, apenas uma minoria $(22 \%)$ não possuía. A nuliparidade tem sido um fator importante associado ao desenvolvimento do câncer de mama ${ }^{6}$. A maioria das participantes relatou ter amamentado por mais de seis meses. É observado que o tempo de amamentação foi relativamente alto entre as participantes, mais do que seis meses. Sendo o preconizado e demostrado como um fator de proteçáo para o câncer de mama quando praticada no mínimo por seis meses ${ }^{9,6}$.

Em relação às características terapêuticas, além do tratamento radioterápico, $70 \%$ das participantes haviam realizado quimioterapia anterior à radioterapia. Com relação ao estadiamento do câncer, foi possível notar que a maioria $(71 \%)$ das pacientes se encontrava em estágios iniciais do câncer de mama classificadas em estádios 0 , I A, II A e II B, o que, consequentemente, pode levar à chance de um melhor prognóstico e tem sido um importante aliado na melhora da taxa de sobrevida. Detectar precocemente o câncer e realizar o rastreamento de populaçôes vulneráveis integram açôes imprescindíveis, para uma melhor assistência a esse público, de maneira a provocar impactos positivos na QVRS ${ }^{35}$.

No que concerne à avaliação da QVRS, por meio do instrumento de EORCT QLQ-C30, apresentada neste estudo, observa-se, nas escalas funcionais, que as médias e desvio-padrão dos escores de função física, desempenho de papel, função cognitiva, função social e emocional apresentaram pontuaçóes elevadas no início do tratamento, sendo considerados em um patamar satisfatório. Podendo ser observado que os escores se elevaram ao longo do tratamento e que, apesar de já estarem classificados como satisfatórios, melhoram ao longo do tempo, finalizando o tratamento radioterápico com melhores médias de escores que os iniciais. Cabe ressaltar que os escores obtidos nas escalas funcionais quanto mais altos e próximos de 100 representam um melhor funcionamento ${ }^{36}$.

A avaliação referente à escala de sintomas do EORTC QLQ-C30 demonstrou que insônia, constipação, fadiga e dor foram os itens de maiores escores, indicando maior intensidade. Resultado semelhante ao encontrado em um estudo que investigou a QVRS e os fatores relacionados para a população iraniana de mulheres com diagnóstico de câncer de mama utilizando o instrumento EORTC QLQ-C30 ${ }^{37}$. Ressalta-se que a insônia $(22,73 \pm 14,89)$ e a fadiga $(19,81 \pm 14,42)$ foram os prevalentes para a escala de sintomas.

Para este estudo, os sintomas de náuseas e vômitos, dispneia, perda de apetite, diarreia e o item dificuldades financeiras apresentaram escore baixo, indicando pouca sintomatologia presente nessas mulheres e pouca interferência financeira advinda do tratamento.

O impacto do tratamento radioterápico em mulheres com câncer de mama também foi investigado em um estudo, no qual foram avaliados aspectos psicológicos, financeiros e sexuais, utilizando-se os instrumentos EORTC QLQ-C30 e QLQ-BR23. Sendo demonstrado que, para as escalas de sintomas, as pontuaçóes mais altas foram apresentadas para insônia $(29,99)$, fadiga $(26,57)$ e dor $(23,05)^{38}$. O que corrobora os resultados da escala 
de sintomas encontrados para este estudo, em que os piores escores foram para esses sintomas, com a inclusão do sintoma de constipaçáo.

Nesta pesquisa, foram comparadas as modificaçôes entre tempos do tratamento radioterápico, evidenciando o efeito da prática integrativa relaxamento com visualização guiada. Foram encontradas diferenças significativas nas escalas do QLQ-C30 em T2 x T3 para a escala funcional nas dimensóes de desempenho de papel $(p=0,0119)$ e função emocional $(p=0,0115)$; para comparação entre $T 1 \times T 3$, houve significância para a função física $(p=0,0360)$, desempenho de papel $(p=0,0450)$ e função emocional $(p=0,0166)$. Para a escala de sintomas, foi encontrada diferença altamente significativa para o sintoma de insônia $(p=0,0001)$ e significância para o domínio dificuldade financeira $(p=0,0467)$. Indicando que a prática de relaxamento com visualização influenciou positivamente esses domínios ao logo do tratamento.

Acrescenta-se um estudo que objetivou determinar a qualidade de vida de pacientes com câncer de mama e detectar as mudanças sofridas após os tratamentos, sendo verificado que a sintomatologia geral das pacientes, a função física e a funcional pioraram significativamente, fato que ocorreu geralmente após o tratamento cirúrgico por conta dos tratamentos recebidos como a quimioterapia, terapias hormonais e radioterapia ${ }^{29}$.

Um estudo randomizado determinou a eficácia da intervenção relaxamento com visualizaçáo guiada na redução da ansiedade e depressão de pacientes com câncer de mama submetidas à braquiterapia. Uma amostra de 66 pacientes foi alocada, 32 para o grupo controle e 34 para o grupo intervençáa, sendo utilizados o instrumento Hospital Anxiety and Depression Scale (HADS) para avaliação da ansiedade e depressão e o instrumento QLCA-AFex para avaliação da qualidade de vida aplicado em três momentos: antes, durante e após a braquiterapia. As pacientes do grupo experimental receberam a sessão de relaxamento por 10 minutos e um CD para continuarem essas sessôes de relaxamento em casa. O grupo intervenção apresentou uma redução estatisticamente significativa da ansiedade $(p=0,008)$, depressáo $(p=0,03)$ e desconforto corporal $(p=0,04)$, e consequentemente melhor qualidade de vida quando comparada ao grupo controle ${ }^{39}$.

Entre as limitaçóes vivenciadas durante o desenvolvimento deste estudo, destaca-se a não randomização, por conta do público limitado de mulheres com câncer de mama. Outra dificuldade encontrada foi a disponibilidade de tempo entre as participantes, visto que muitas dependiam do transporte para comparecer ao serviço, isso gerava uma dificuldade no atendimento. Uma das opçóes para sanar essas dificuldades seria estender o período de coleta de dados, tornando possível o processo de randomização e a ampliação do público estudado.

\section{CONCLUSÃO}

O uso de técnicas de relaxamento com visualizaçáo guiada mostrou-se eficaz na melhora da QVRS.

As médias de escores para o EGS/QV melhoraram ao longo do tratamento radioterápico, indicando que essas mulheres consideraram sua saúde geral e sua QVRS melhor durante a última semana da radioterapia. Os escores relacionados às escalas funcionais foram satisfatórios, porém melhoraram ao longo das avaliaçóes, apresentando o melhor escore ao final do tratamento. Apenas a funçáo social, que envolve a relaçáo familiar e as atividades sociais, apresentou oscilaçóes entre as médias de escore, sendo todos classificados como satisfatórios.

Em relação à escala de sintomas, o prevalente foi a insônia, seguido pela constipaçáo e fadiga, sendo observado que a insônia apresentou pior escore na avaliação mediana e retornou ao escore inicial ao final do tratamento. Os sintomas de constipação e fadiga apresentaram menor intensidade quando comparados à primeira avaliação. Em relação aos tempos de avaliação, a maior significância entre as escalas foi encontrada entre T1 e T3 do tratamento radioterápico para o EGS/QV, função física, desempenho de papel, insônia e dificuldades financeiras.

Diante do atual cenário, com o aumento das doenças crônico-degenerativas, principalmente o câncer, torna-se imprescindível que o profissional enfermeiro volte seu olhar para os pacientes em tratamentos oncológicos, com intuito de redução dos efeitos colaterais advindos do tratamento e na melhora QVRS. O uso de técnicas de relaxamento com visualização guiada mostrou-se eficaz e apresenta benefícios por ser de baixo custo, utiliza poucos recursos tecnológicos e pode ser aplicado por profissionais treinados, contribuindo em todos os momentos do itinerário terapêutico.

Fica evidenciado que poucos estudos com ênfase em mulheres em tratamento radioterápico foram identificados, demostrando a importância de que mais estudos com esse grupo sejam desenvolvidos, visto que o tratamento radioterápico traz efeitos colaterais durante seu processo terapêutico que tendem a causar prejuízos à QVRS.

\section{CONTRIBUIÇÕES}

Todos os autores contribuíram substancialmente na concepção e no planejamento do estudo; na obtenção, análise e/ou interpretação dos dados; assim como na 
redação e/ou revisão crítica; e aprovaram a versão final a ser publicada.

\section{DECLARAÇÃO DE CONFLITO DE INTERESSES}

Nada a declarar.

\section{FONTES DE FINANCIAMENTO}

Coordenação de Aperfeiçoamento de Pessoal de Nível Superior - Brasil (Capes). Código de Financiamento 001.

\section{REFERÊNCIAS}

1. Wild CP, Weiderpass E, Stewart BW, editors. World Cancer Report: cancer research for cancer prevention [Internet]. Lyon: International Agency for Research on Cancer; 2020 [cited 2020 mar 21]. Available from: https://www.iccp-portal.org/system/ files/resources/IARC\%20 World\%20Cancer $\% 20$ Report\%202020.pdf

2. Siegel RL, Miller KD, Jemal A. Cancer Statistics, 2020. CA Cancer J Clin. 2020;70(1):7-30. doi: https://doi. org/10.3322/caac. 21590

3. Marta GN, Hanna SA, Martella E, et al. Câncer de mama estádio inicial e radioterapia: atualização. Rev Assoc Med Bras. 2011;57(4):468-74. doi: https://doi.org/10.1590/ S0104-42302011000400024

4. Instituto Nacional de Câncer José Alencar Gomes da Silva. Estimativa 2020: incidência de câncer no Brasil [Internet]. Rio de Janeiro: INCA; 2019 [acesso 2020 jul 9]. Disponível em: https://www.inca.gov.br/sites/ufu. sti.inca.local/files//media/document//estimativa-2020incidencia-de-cancer-no-brasil.pdf

5. Ministério da Saúde (BR), Secretaria de Atenção à Saúde. Portaria n.19, de 19 de julho de 2018. Aprova as Diretrizes Diagnósticas e Terapêuticas do Carcinoma de Mama [Internet]. Diário Oficial da União. 2018 jul 16 [acesso 2020 abr 12]. Edição 135, Seção 1:59. Disponível em: https://www.in.gov.br/materia/-/asset_publisher/ Kujrw0TZC2Mb/content/id/31547238

6. Instituto Nacional de Câncer José Alencar Gomes da Silva [Internet]. Rio de Janeiro: INCA; [data desconhecida]. Tipos de câncer: câncer de mama; 2020 [modificado 2021 set 2; acesso 2020 set 30]. Disponível em: https:// www.inca.gov.br/tipos-de-cancer/cancer-de-mama

7. Johns C, Seav SM, Dominick SA, et al. Informing hot flash treatment decisions for breast cancer survivors: a systematic review of randomized trials comparing active interventions. Breast Cancer Res Treat. 2016;156(3):41526. doi: http://doi.org/10.1007/s10549-016-3765-4

8. Haque R, Shi J, Schottinger JE. Tamoxifen and antidepressant drug interaction in a cohort of 16,887 breast cancer survivors. J Natl Cancer Inst. 2016;108(3):djv337. doi: http://doi.org/10.1093/jnci/djv337

9. Vieira SC, Reis CA, Silva DFR, et al., editores. Câncer de mama: consenso da Sociedade Brasileira de Mastologia: regional Piauí - 2017 [Internet]. Teresina, PI: EDUFPI; 2017 [acesso 2020 jul 30]. Disponível em: https:// sbmastologia.com.br/wp-content/uploads/2021/08/ Ca\%CC\%82ncer-de-Mama-Consenso-da-SBMRegional-Piaui\%CC\%81-2017.pdf

10. Instituto Nacional de Câncer José Alencar Gomes da Silva [Internet]. Rio de Janeiro: INCA; [data desconhecida]. Câncer: estadiamento; [modificado 2021 ago 4; acesso 2020 jul 30]. Disponível em: https://www.inca.gov.br/ estadiamento

11. American Cancer Society [Internet]. Atlanta (GA): American Cancer Society; c2021 Breast Cancer Screening Guidelines; [cited 2020 fev 23]. Available from: https:// www.cancer.org/health-care-professionals/americancancer-society-prevention-early-detection-guidelines/ breast-cancer-screening-guidelines.html

12. Ministério da Saúde (BR); Comissão Nacional de Incorporação de Tecnologias no SUS. Radioterapia intraoperatória de tumores de mama [Internet]. Brasília, DF: CONITEC; 2016 [acesso 2020 mar 28]. Disponível em: http://conitec.gov.br/images/Relatorios/2016/ Relatorio_Radioterapia_TumorMama_final.pdf

13. Rocha DM, Pedrosa AO, Oliveira AC, et al. Evidências científicas sobre os fatores associados à qualidade de vida de pacientes com radiodermatite. Rev Gaúcha Enferm. 2018;39:e2017-0224. doi: https://doi. org/10.1590/1983-1447.2018.2017-0224

14. Bahia JC, Lima CM, Oliveira MM, et al. Fadiga em mulheres com câncer de mama submetidas à radioterapia. Rev Bras Cancerol. 2019;65(2):e-09089. doi: https://doi. org/10.32635/2176-9745.RBC.2019v65n2.89

15. Oliveira KFP, Lima LDAC, Carvalho SML. Morbidades após tratamento do câncer de mama. An Fac Med Olinda [Internet]. 2018 [acesso 2020 set 9];2(2):91-95. Disponível em: https://afmo.emnuvens.com.br/afmo/ article/view/43/40

16. Brownlee Z, Garg R, Listo M, et al. Late complications of radiation therapy for breast cancer: evolution in techniques and risk over time. Gland Surg. 2018;7(4):371-8. doi: https://doi.org/10.21037/gs.2018.01.05

17. Paula JM, Sawada NO. Health-related quality of life of cancer patients undergoing radiotherapy. Rev Rene. 2015;16(1):106-13. doi: https://doi.org/10.15253/21756783.2015000100014

18. Queiroz FA, Pace AE, Santos CB. Adaptação cultural e validação do instrumento Diabetes - 39 (D-39): versão para brasileiros com diabetes mellitus tipo 2 - fase 1 . Rev Latino-Am Enfermagem. 2009;17(5):708-15. doi: https://doi.org/10.1590/S0104-11692009000500018 
19. Wortmann JK, Bremer A, Eich HT, et al. Use of complementary and alternative medicine by patients with cancer: a cross-sectional study at different points of cancer care. Med Oncol. 2016;33(7):78. doi: http:// doi.org/10.1007/s12032-016-0790-4

20. Leite PS, Marques IMM, Clementino CFL, et al. Uso de práticas integrativas e complementares no paciente oncológico. Id on Line Rev Psicol. 2018;12(40):39. doi: https://doi.org/10.14295/idonline.v12i40.1092

21. Lyman GH, Greenlee H, Bohlke K, et al. Integrative therapies during and after breast cancer treatment: Asco endorsement of the sio clinical practice guideline summary. J Clin Oncol. 2018;36(25):2647-55. doi: http://doi.org/10.1200/JCO.2018.79.2721

22. Maindet C, Burnod A, Minello C, et al. Strategies of complementary and integrative therapies in cancer-related pain-attaining exhaustive cancer pain management. Support Care Cancer. 2019;27(8):3119-32. doi: https:// doi.org/10.1007/s00520-019-04829-7

23. Grant SJ, Hunter J, Seely D, et al. Integrative oncology: international perspectives. Integr Cancer Ther. 2019;18:1534735418823266. doi: https://doi. org/10.1177/1534735418823266

24. Metin ZG, Ejem D, Dionne-Odom JN, et al. Mindbody interventions for individuals with heart failure: a systematic review of randomized trials. J Card Fail. 2018;24(3):186-201. doi: https://doi.org/10.1016/j. cardfail.2017.09.008

25. Ministério da Saúde (BR). Portaria n. 702, de 21 de março de 2018. Altera a Portaria de Consolidaçáo no $2 /$ GM/MS, de 28 de setembro de 2017, para incluir novas práticas na Política Nacional de Práticas Integrativas e Complementares - PNPIC [Internet]. Diário Oficial da Uniāo. 2018 [acesso 2020 abr 12]; Seção1:74. Disponível em: http://bvsms.saude.gov.br/bvs/saudelegis/gm/2018/ prt0702_22_03_2018.html

26. Brabo EP, Paschoal MEM, Biasoli I, et al. Brazilian version of the QLQ-LC13 lung cancer module of the European Organization for Research and Treatment of Cancer: preliminary reliability and validity report. Qual Life Res. 2006;15(9):1519-24. doi: https://doi. org/10.1007/s11136-006-0009-9

27. Aaronson NK, Ahmedzai S, Bergman B, et al. The European Organization for Research and Treatment of Cancer QLQ-C30: a quality-of-Life instrument for use in international clinical trials in oncology. J Natl Cancer Inst. 1993;85(5):365-76. doi: http://doi.org/10.1093/ jnci/85.5.365

28. Soares PBM, Quirino Filho S, Souza PW, et al. Características das mulheres com câncer de mama assistidas em serviços de referência do norte de minas gerais. Rev Bras Epidemiol. 2012;15(3):595-
604. doi: https://doi.org/10.1590/S1415790X2012000300013

29. Villar RR, Fernández SP, Garea CC, et al. Qualidade de vida e ansiedade em mulheres com câncer de mama antes e depois do tratamento. Rev Latino-Am Enfermagem. 2017;25:e2958. doi: https://doi.org/10.1590/15188345.2258.2958

30. Bonotto GM, Mendoza-Sassi RA, Susin LRO. Conhecimento dos fatores de risco modificáveis para doença cardiovascular entre mulheres e seus fatores associados: um estudo de base populacional. Ciênc Saúde Colet. 2016;21(1):293-302. doi: https://doi. org/10.1590/1413-81232015211.07232015

31. Batiston AP, Tamaki EM, Souza LA, et al. Conhecimento e prática sobre os fatores de risco para o câncer de mama entre mulheres de 40 a 69 anos. Rev Bras Saude Mater Infant. 2011;11(2):163-71. doi: http://doi.org/10.1590/ S1519-38292011000200007

32. Leite MAC, Nogueira DA, Terra FS. Avaliação da autoestima em pacientes oncológicos submetidos a tratamento quimioterápico. Rev Latino-Am Enfermagem. 2015;23(6):1082-9. doi: http://doi.org/10.1590/01041169.0575 .2652

33. Guerrero GP, Zago MMF, Sawada NO, et al. Relaçáo entre espiritualidade e câncer: perspectiva do paciente. Rev Bras Enferm. 2011;64(1):53-9. doi: https://doi. org/10.1590/S0034-71672011000100008

34. Ribeiro GS, Campos CS, Anjos ACY. Spirituality and religion as resources for confronting breast cancer. R Pesq Cuid Fundam Online. 2019;11(4):849-56. doi: http:// doi.org/10.9789/2175-5361.2019.v11i4.849-856

35. Fortes RC, Haack A, Frazão ERP. Qualidade de vida de mulheres com câncer de mama: uma revisão integrativa. Com Ciências Saúde. 2018;29(4):218-25. doi: https:// doi.org/10.51723/ccs.v29i04.395

36. Hinz A, Singer S, Brähler E. European reference values for the quality of life questionnaire eortc qlq-c30: results of a german investigation and a summarizing analysis of six european general population normative studies. Acta Oncol. 2014;53(7):958-65. doi:http://doi.org/10.3109/ 0284186X.2013.879998

37. Shandiz FH, Karimi FZ, Anbaran ZK, et al. Investigating the quality of life and the related factors in iranian women with breast cancer. Asian Pac J Cancer Prev. 2017;18(8):2089-92. doi: http://doi.org/10.22034/ APJCP.2017.18.8.2089

38. Sharma N, Purkayastha A. Impact of radiotherapy on psychological, financial, and sexual aspects in postmastectomy carcinoma breast patients: a prospective study and management. Asia Pac J Oncol Nurs. 2017;4(1):69-76. doi: http://doi.org/10.4103/23475625.199075 
39. León-Pizarro C, Gich I, Barthe E, et al. A randomized trial of the effect of training in relaxation and guided imagery techniques in improving psychological and quality-of-life índices for gynecologic and breast brachytherapy patients. Psychooncology. 2007;16(11):971-9. doi: http://doi. org/10.1002/pon. 1171

Recebido em 18/11/2020

Aprovado em 26/4/2021 